\title{
Classification of approximately inner automorphisms of subfactors
}

\author{
YASUYUKI KAWAHIGASHI \\ Department of Mathematical Sciences \\ University of Tokyo, Komaba, Tokyo, 153, JAPAN \\ e-mail: yasuyuki@ms.u-tokyo.ac.jp \\ Dedicated to Professor Hikosaburo Komatsu on his sixtieth birthday
}

\begin{abstract}
For classification of approximately inner automorphisms of subfactors, we introduce a new invariant, a higher obstruction. From an algebraic viewpoint, this can be regarded as a generalization of the Connes obstruction, and from an analytic viewpoint, this can be regarded as a generalization of the Jones invariant $\kappa$. We have two classification theorems for approximately inner automorphisms of strongly amenable subfactors with known invariants and this new one. In particular, our theorems give a complete classification of automorphisms, up to outer conjugacy, of AFD subfactors of type $\mathrm{II}_{1}$ with index less than four except for one special case for $A_{4 n-1}$ and $E_{6}$.
\end{abstract}

\section{Introduction}

For single factors, classification problems of automorphisms and group actions on injective factors have caught much attention since the fundamental work of A. Connes [7], and many successful classification theories have been available. In subfactor theory initiated by V. F. R. Jones [19], a systematic study of automorphisms of subfactors was initiated by P. H. Loi in [27]. Loi's main motivation was a study of subfactors of type $\mathrm{III}_{\lambda}(0<\lambda<1)$, as one of the main applications of the Connes classification of automorphisms of type II factors was a classification of factors of type $\mathrm{III}_{\lambda}$.

In subfactor theory, S. Popa has obtained a powerful classification result of automorphisms of subfactors in [33] in the following form: Properly outer actions of a discrete amenable group on a strongly amenable subfactors of type $\mathrm{II}_{1}$ are classified by Loi's invariants up to cocycle conjugacy. (This is, of course, based on Popa's "ultimate" analytic classification theorem in [31] that a strongly amenable subfactors of type $\mathrm{II}_{1}$ has the generating property. - The terminologies in these theorems will be explained below.) This deep theorem solves the original problem of Loi of classification of strongly amenable subfactors of type $\mathrm{III}_{\lambda}$ completely. Subfactors, however, often have interesting automorphisms with trivial Loi invariant, as shown 
in our previous work [11], [12], [21], [22]. Thus we expect an interesting classification theory for automorphisms of subfactors which are not classified by Loi's invariant. Our aim in this paper is to get certain classification results for these automorphisms with our new invariants. By the results in [27, Theorem 5.4] and [31], we know that the class of automorphisms of strongly amenable subfactors with trivial Loi invariant is exactly that of approximately inner automorphisms. So our classification results here are for approximately inner automorphisms. (Precise definitions of these notions will be given below.)

Before going into our theory, we first point out that a "complete classification" of automorphisms of subfactors in a sense can be obtained rather easily for strongly amenable subfactors with our previous results in [23]. (This approach was first suggested by S. Popa. T. Ceccherini [4] got the first classification result on this approach.)

That is, for a strongly amenable subfactor $N \subset M$ and an automorphism $\alpha$ of $M$ with $\alpha(N)=N$, we study the following commuting squares of $\mathrm{II}_{1}$ factors.

$$
\begin{aligned}
& \left\{\left(\begin{array}{ccc}
x & 0 & 0 \\
0 & \alpha(x) & 0 \\
0 & 0 & \alpha(x)
\end{array}\right) \mid x \in N\right\} \subset\left\{\left(\begin{array}{ccc}
x_{11} & x_{12} & x_{13} \\
x_{21} & x_{22} & x_{23} \\
x_{31} & x_{32} & x_{33}
\end{array}\right) \mid x_{i j} \in N\right\} \\
& \left\{\left(\begin{array}{ccc}
x & 0 & 0 \\
0 & \alpha(x) & 0 \\
0 & 0 & \alpha(x)
\end{array}\right) \mid x \in M\right\} \subset\left\{\left(\begin{array}{lll}
x_{11} & x_{12} & x_{13} \\
x_{21} & x_{22} & x_{23} \\
x_{31} & x_{32} & x_{33}
\end{array}\right) \mid x_{i j} \in M\right\}
\end{aligned}
$$

Then it is easy to see that an outer conjugacy classification of $\alpha$ is equivalent to classification of this commuting squares. This type of commuting squares has been classified completely by us with the standard invariant as in [23, Theorem 3.4]. Furthermore, we are often interested in subfactors with finite depth, and in such a case, any aperiodic automorphism gives a centrally free action of $\mathbf{Z}$ by [20, Proposition 3.1], [27, Proposition 4.4]. Then they are classified by Loi's invariant by Popa's classification theorem. Thus the remaining case involves only automorphisms of finite order. Then the above classification based on [23] is really reduced to a problem of finite combinatorics.

The above "complete solution" is, however, not satisfactory from the viewpoint of classical classification results in [7], [9], [10], and so on. This is because it is very hard to write down a list of all the outer conjugacy classes for a given subfactor in the above approach. That is, although we have a complete invariant, its computation is very hard and we practically cannot determine the range of the invariant. (Even in the case of the Jones subfactors of type $A_{n}$ in [19], this problem of listing is already hard enough.) So we hope to get a more concrete classification result being parallel to the classical classification results along the line of Connes [7], [9], [10].

We will introduce such concrete invariants here and show that they give a complete classification for a nice class of subfactors such as the Jones subfactors of type $A_{4 n-3}$ and the Wenzl subfactors with indices converging to 9. (See Examples 6.2 and 6.7.) These subfactors give the first examples of a complete classification of automorphisms 
of subfactors which is different from the Connes classification of automorphisms of single factors in [7].

In Section 2, we introduce our new invariants from an algebraic viewpoint. We will show a first classification theorem for approximately inner automorphisms of subfactors in Section 3. In Section 4, we define the invariants from an analytic viewpoint and prove that the algebraic and analytic definitions give essentially same invariants for strongly amenable subfactors. This coincidence generalizes a theorem in [24, Theorem 3.4] where we identified an obstruction to flatness in the quantum $S U(n)_{k}$ orbifold construction with the relative Jones invariant $\kappa$. We then get another classification theorem in Section 5 for the case not covered by the theorem in Section 3. In the last Section 6, we work out the classification on concrete examples of subfactors and give a philosophical reason our higher obstruction should be the last missing invariant for classification of automorphisms of subfactors, based on an analogy to classification of automorphisms of injective factors of type III. We also show that our Theorems give a complete classification of (approximately inner) automorphisms, up to outer conjugacy, of a large subclass of the Hecke algebra subfactors of Wenzl [37].

A part of this work was done at the Fields Institute for Research in Mathematical Sciences in the spring of 1995 and the author thanks the Institute for financial supports and hospitality during the stay. The author also acknowledges financial supports from the Inamori Foundation and the Sumitomo Foundation during this research. He thanks V. F. R. Jones for his question on [24] mentioned here after Theorem 4.1 and S. Goto for pointing out typographical errors and ambiguous points in the original draft of this paper.

\section{New invariants - an algebraic approach -}

We now describe our new invariants. Let $N \subset M$ be a subfactor of type $\mathrm{II}_{1}$. For simplicity, we also assume in this paper that $M$ [resp. $\left.M_{1}\right]$ has no non-trivial normalizer of $N$ [resp. $M]$. (If we have non-trivial normalizers, then the subfactor has a "classical" intermediate subfactor coming from an action of a finite group. Our philosophy is that an interesting "quantum" subfactor does not have such an intermediate subfactor.) Then $\alpha \in \operatorname{Aut}(M, N)$ is inner on $M$ if and only if it is inner on $N$, so it makes sense to have an outer period $p_{o}(\alpha)$.

Let $\operatorname{Aut}(M, N)=\{\alpha \in \operatorname{Aut}(M, N) \mid \alpha(N)=N\}$. We say that $\alpha, \beta \in \operatorname{Aut}(M, N)$ are outer conjugate if there exist a unitary $u \in N$ and $\theta \in \operatorname{Aut}(M, N)$ such that $($ Ad $u) \cdot \alpha=\theta \cdot \beta \cdot \theta^{-1}$.

Consider any $\alpha \in \operatorname{Aut}(M, N)$. Choda-Kosaki gave a definition of strong outerness of automorphisms of subfactors in [6, Definition 1], which is the same as proper outerness of Popa in [33, 1.5]. That is, $\alpha$ is not strongly outer if there exists a nonzero $a \in M_{k}$ in the Jones tower such that we have $\alpha(x) a=a x$ for all $x \in M$. We define the strongly outer period $p_{s}(\alpha) \in \mathbf{N}$ of $\alpha$ by

$$
p_{s}(\alpha) \mathbf{Z}=\left\{n \in \mathbf{Z} \mid \alpha^{n} \text { is not strongly outer. }\right\} .
$$


The characterization of not strongly outer automorphisms by Choda-Kosaki [6, Theorem 2], [26, Theorem 3] is as follows. An automorphism $\alpha$ of $N \subset M$ is not strongly outer if and only if it appears as a vertex of the dual principal graph (as an $M-M$ bimodule). (Also see [13, Theorem 2.2].)

Suppose now $p_{s}(\alpha)>0$ and set $p=p_{s}(\alpha)$. The power $\alpha^{p}$ gives an element in

$$
\{\alpha \in \operatorname{Aut}(M, N) \mid \alpha \text { is not strongly outer. }\} / \operatorname{Int}(M, N),
$$

where $\operatorname{Int}(M, N)=\{$ Ad $u \mid u \in \mathcal{U}(N)\}$. We write $\nu_{\text {alg }}(\alpha)$ for the orbit of the image of $\alpha^{p}$ under the action of $\operatorname{Aut}(M, N)$ given by $\alpha^{p} \mapsto \theta \cdot \alpha^{p} \cdot \theta^{-1}$ for $\theta \in \operatorname{Aut}(M, N)$ and call it an algebraic $\nu$ invariant. This is clearly an outer conjugacy invariant. (See [15, Example 2.9] for an example where we have to take this orbit to get an outer conjugacy invariant.) In many interesting cases, this orbit turns out to consist of a single element as we will see in Section 6. This invariant can be regarded as an analogue of the modular invariant of Sutherland-Takesaki [35] and the symbol $\nu$ comes from this analogy. See Section 6 for more on this analogy.

We know that there exist $k \geq 0$ and $a \in M_{k} \backslash\{0\}$ such that $\alpha^{p}(x) a=a x$ for all $x \in M$. We fix a minimal $k$ with this property here. Let

$$
I_{\alpha}=\left\{a \in M_{k} \mid \alpha^{p}(x) a=a x, \text { for all } x \in M\right\} .
$$

This is a Hilbert space with respect to the inner product arising from the trace. It is clear that $\alpha$ gives a unitary action $U_{\alpha}$ on $I_{\alpha}$. On the space of unitary matrices in a fixed dimension, the unitary matrices themselves acts by adjoint. We define $\gamma_{h}(\alpha)$ to be the orbit of $U_{\alpha}$ under this action. We call $\gamma_{h}(\alpha)$ a higher obstruction of $\alpha$.

Theorem 2.1 The higher obstruction $\gamma_{h}(\alpha)$ is an outer conjugacy invariant of $\alpha$.

Proof It is clear that $\gamma_{h}(\alpha)$ is a conjugacy invariant, by definition.

Let $u$ be a unitary in $N$, and set $\tilde{\alpha}=(\operatorname{Ad} u) \cdot \alpha$. Then it is trivial that the strongly outer period of $\tilde{\alpha}$ is also $p$, and we have

$$
\tilde{\alpha}^{p}=\operatorname{Ad}\left(u \cdot \alpha(u) \cdots \alpha^{p-1}(u)\right) \cdot \alpha^{p} .
$$

Set $v=u \cdot \alpha(u) \cdots \alpha^{p-1}(u)$. Then we have $u \alpha(v)=v \alpha^{p}(u)$. We also have that $\alpha^{p}(x) a=a x$ if and only of $\tilde{\alpha}^{p}(x) v a=\operatorname{vax}$ for all $x \in M$. This gives a natural isomorphism between $I_{\alpha}$ and $I_{\tilde{\alpha}}$. To prove $\gamma_{h}(\alpha)=\gamma_{h}(\tilde{\alpha})$, we need to prove that $\tilde{\alpha}(v a)=v \alpha(a)$ for all $a \in I_{\alpha}$. We see this identity by

$$
\tilde{\alpha}(v a)=u \alpha(v) \alpha(a) u^{*}=v \alpha^{p}(u) \alpha(a) u^{*}=v \alpha(a) u u^{*}=v \alpha(a),
$$

where we used the property $\alpha(a) \in I_{\alpha}$.

Q.E.D.

We next recall the definition of the Loi invariant $\Phi(\alpha)$ of $\alpha \in \operatorname{Aut}(M, N)$. As in $[27, \S 5]$, we can extend $\alpha$ to the Jones tower $\left\{M_{k}\right\}$ canonically by setting $\alpha\left(e_{k}\right)=e_{k}$. Then $\Phi(\alpha)$ is defined as an action of this extension on the commuting squares of the higher relative commutant $\left\{M^{\prime} \cap M_{k} \subset N^{\prime} \cap M_{k}\right\}_{k}$. The higher obstruction takes a simple form of a scalar if the automorphism has a trivial Loi invariant. This will play a key role in the classification theorem later. 
Theorem 2.2 If $\Phi(\alpha)$ is trivial, then $\gamma_{h}(\alpha) \in \mathbf{C}$. Furthermore, for any l, if $a \in M_{l}$ satisfies $\alpha^{p}(x) a=$ ax for all $x \in M$, then we get $\alpha(a)=\gamma_{h}(\alpha) a$.

Proof We use notations and arguments of [13, Theorem 2.2], which is based on [6], [26]. We know that $I_{\alpha}$ is given by the images of $1 \in M_{\alpha^{p}}$ by $\xi^{*}$ for all $\xi \in$ $\operatorname{Hom}\left({ }_{M} M_{k M},{ }_{M} M_{\alpha^{p} M}\right)$. Because $U_{\alpha}$ is unitary on $I_{\alpha}$, we can choose an orthonormal system $\left\{a_{j}\right\}_{j} \subset I_{\alpha}$ with $U_{\alpha} a_{j}=c_{j} a_{j}$ for some scalars $c_{j}$. Then we may assume that each $a_{j}$ is represented as $\xi_{j}^{*}(1)$ for an orthonormal family $\left\{\xi_{j}\right\}_{j} \subset$ $\operatorname{Hom}\left({ }_{M} M_{k M},{ }_{M} M_{\alpha^{p} M}\right)$.

Then $\left\{\xi_{j}^{*} \xi_{k}\right\}_{j k}$ gives a system of matrix units in $M^{\prime} \cap M_{2 k}$ (for the summand corresponding to $\left.\alpha^{p}\right)$. Because the Loi invariant of $\alpha$ is trivial, we have $\alpha\left(\xi_{j}^{*} \xi_{k}\right)=\xi_{j}^{*} \xi_{k}$. It is also easy to see $a_{j}=\xi_{j}^{*} \xi_{k} a_{k}$, so by applying $\alpha$ to the both hand sides, we get $c_{j} a_{j}=c_{k} \xi_{j}^{*} \xi_{k} a_{k}$. This implies $c_{j}=c_{k}$ for all $j, k$, and hence $U_{\alpha}$ is a scalar.

For $l>k$, we can embed $I_{\alpha}$ into $M_{l}$, and repeat the above argument for $M_{l}$ to get the conclusion in the second half of the Theorem.

Q.E.D.

We also prepare a lemma we will need in the next section.

Lemma 2.3 Suppose that $p_{s}(\alpha)>0$ and that the order $n$ of $\nu_{\mathrm{alg}}(\alpha)$ in the group $\operatorname{Aut}(M, N) / \operatorname{Int}(M, N)$ is finite. (The finiteness of $n$ automatically holds if $N \subset M$ has finite depth by [6, Theorem 2].) We also assume that $\Phi(\alpha)$ is trivial. Then the outer period of $\alpha$ is np and the Connes obstruction of $\alpha$ is $\gamma_{h}(\alpha)^{n}$, where $p=p_{s}(\alpha)$.

Proof It is trivial that the outer period of $\alpha$ is $n p$.

Let $u$ be a unitary in $N$ with $\alpha^{n p}=$ Ad $u$. By Theorem 2.2, the higher obstruction $\gamma_{h}(\alpha)$ is scalar, and we have $a \in M_{k} \backslash\{0\}$ with $\alpha^{p}(x) a=a x$ for all $x \in M$ and $\alpha(a)=\gamma_{h}(\alpha) a$, where $k$ is chosen to be minimal.

The argument in [13, Theorem 2.2] shows that $a \otimes_{M} a \otimes_{M} \cdots \otimes_{M} a$ is given by $\xi^{*}(1)$ for some non-zero intertwiner $\xi$, and thus $a \otimes_{M} a \otimes_{M} \cdots \otimes_{M} a \neq 0$. Let $\tilde{a} \in M_{n k}$ be the corresponding element in the canonical isomorphism between $M_{k} \otimes_{M} M_{k} \otimes_{M} \cdots \otimes_{M} M_{k}$ and $M_{n k}$ as $M-M$ bimodules. That is, we have $\tilde{a}=a f_{1} a f_{2} a \cdots a f_{n-1} a$, where $f_{m}$ is a product of a positive scalar and the Jones projections $e_{j}$ 's as in [30]. Then we have $\tilde{a} \neq 0, \alpha^{n p}(x) \tilde{a}=\tilde{a} x$ for all $x \in M$, and $\alpha(\tilde{a})=\gamma_{h}^{n}(\alpha) \tilde{a}$. We then have $u x u^{*} \tilde{a}=\alpha^{n p}(x) \tilde{a}=\tilde{a} x$ for all $x \in M$. This means $u^{*} \tilde{a} \in M^{\prime} \cap M_{n k}$. Because the Loi invariant of $\alpha$ is trivial, we get $\alpha\left(u^{*} \tilde{a}\right)=u^{*} \tilde{a}$. It then follows that $\alpha(u)=\gamma_{h}(\alpha)^{n} u$, as desired.

Q.E.D.

The above lemma implies $\gamma_{h}(\alpha)^{p n^{2}}=1$, in particular.

Note that if $N=M$, then $p_{s}(\alpha)$ is the outer period $p_{o}(\alpha)$ of $\alpha$ and the higher obstruction $\gamma_{h}(a)$ is same as the Connes obstruction of $\alpha$ in [10].

Also note that if the $*$-vertex of the dual principal graph of $N \subset M$ is the only vertex with Perron-Frobenius weight 1 , then $p_{s}$ and $\gamma_{h}$ of $\alpha \in \operatorname{Aut}(M, N)$ are same as $p_{o}$ and $\gamma$ of $\alpha \in \operatorname{Aut}(N)$ (or $\left.\alpha \in \operatorname{Aut}(M)\right)$ by [6, Theorem 2]. 


\section{The first classification theorem}

We recall some notations from [27] as follows.

$$
\begin{aligned}
\overline{\operatorname{Int}}(M, N) & =\text { closure of } \operatorname{Int}(M, N), \\
\operatorname{Ct}(M, N) & =\left\{\alpha \in \operatorname{Aut}(M, N) \mid \alpha=\mathrm{id} \text { on } N^{\omega} \cap M^{\prime}\right\},
\end{aligned}
$$

where $\omega$ is any free ultrafilter over $\mathbf{N}$. (The definition of $\operatorname{Ct}(M, N)$ does not depend on a choice of $\omega$.$) Automorphisms in \overline{\operatorname{Int}}(M, N)$ and $\operatorname{Ct}(M, N)$ are called approximately inner and centrally trivial, respectively.

We here give the first classification theorem for strongly amenable subfactors $N \subset M$. Recall that $\alpha \in \operatorname{Aut}(M, N)$ is approximately inner if and only if $\Phi(\alpha)$ is trivial by [27, Theorem 5.4] and [31] and that $\alpha \in \operatorname{Aut}(M, N)$ is centrally trivial if and only if it is not strongly outer by [32, section 4].

For approximately inner automorphisms, we have the following classification theorem first.

Theorem 3.1 Let $N \subset M$ be a strongly amenable subfactor of type $I I_{1}$. Suppose that $\alpha, \beta \in \operatorname{Aut}(M, N)$ have trivial Loi invariants and we have the following properties.

1. $p_{s}(\alpha)=p_{s}(\beta)=p$.

2. $\nu_{\text {alg }}(\alpha)=\nu_{\text {alg }}(\beta)$.

3. $\gamma_{h}(\alpha)=\gamma_{h}(\beta)$.

4. $p_{o}(\sigma)>0$ for $\sigma=\alpha^{p}$.

5. $\gamma_{h}(\sigma)=1$.

Then $\alpha$ and $\beta$ are outer conjugate.

Proof The case $p=0$ follows exactly from Popa's classification theorem in [33], so we may assume $p>0$ here. We may also assume that $\beta^{p}=\sigma \operatorname{modulo} \operatorname{Int}(M, N)$.

Set $p=p_{s}$ and $\gamma=\gamma_{h}$. Consider an automorphism $s_{p}^{\bar{\gamma}} \otimes \alpha$ on $R \otimes N \subset R \otimes$ $M$, where $R$ is an approximately finite dimensional (AFD) $\mathrm{II}_{1}$ factor and $s_{p}^{\bar{\gamma}}$ is the automorphism defined by Connes in [10]. This can be regarded as an automorphism of $N \subset M$ because $R \otimes N \subset R \otimes M$ is isomorphic to $N \subset M$. (Note that these two subfactors have the same higher relative commutants, so we can appeal to the general classification theorem of Popa [31]. This is the property called a relative McDuff splitting — see [2].)

Then it is easy to see $p_{s}\left(s_{p}^{\bar{\gamma}} \otimes \alpha\right)=p$ and $\gamma_{h}\left(s_{p}^{\bar{\gamma}} \otimes \alpha\right)=1$. (See [22, Proposition 4.6] or [26, Corollary 7] for the first equality.) Then $\alpha$ is outer conjugate to $s_{p}^{\gamma} \otimes s_{p}^{\bar{\gamma}} \otimes \alpha$ as in [7, Theorem 2.3.1], so it is enough to prove that $s_{p}^{\bar{\gamma}} \otimes \alpha$ and $s_{p}^{\bar{\gamma}} \otimes \beta$ are outer conjugate. That is, we may assume that $\gamma=1$ from the beginning. Then the Connes obstructions of $\alpha$ and $\beta$ are both 1 by Lemma 2.3. Thus we may assume that both $\alpha$ and $\beta$ give outer actions of $\mathbf{Z}_{n p}$. Then the simultaneous crossed products 
$N \rtimes_{\alpha} \mathbf{Z}_{n p} \subset M \rtimes_{\alpha} \mathbf{Z}_{n p}$ give an orbifold subfactor in the sense of [15, Definition 2.10]. (See [11], [13], [14], [15], [21], [39], [40] for the orbifold subfactors.)

We claim that the dual actions $\hat{\alpha}$ and $\hat{\beta}$ of $\mathbf{Z}_{n p}$ are both strongly outer. This is proved for $\alpha$ as follows. Suppose $a=\sum_{j=0}^{n p-1} a_{j} u^{j} \in M_{k} \rtimes_{\alpha} \mathbf{Z}_{n p}$ satisfies

$$
\begin{aligned}
\sum_{j=0}^{n p-1} \hat{\alpha}^{l}(x) a_{j} u^{j} & =\sum_{j=0}^{n p-1} a_{j} u^{j} x, \quad \text { for all } x \in M, \\
\sum_{j=0}^{n p-1} \hat{\alpha}^{l}(u) a_{j} u^{j} & =\sum_{j=0}^{n p-1} a_{j} u^{j} u,
\end{aligned}
$$

for some $l$ with $0<l<n p$, where $u$ is the implementing unitary for the crossed product by the action $\alpha$. The first identity implies that $x a_{j}=a_{j} \alpha^{j}(x)$ for all $x \in$ $M$, which means $a_{j}=0$ unless $j$ is a multiple of $p$. The second identity implies $e^{2 \pi i l / n p} \alpha\left(a_{j}\right)=a_{j}$. Then the assumption $\gamma_{h}(a)=1$ gives $a_{j}=0$ for all $j$, and thus the dual action $\hat{\alpha}$ is strongly outer. This, together with Takesaki duality and Theorem 6.1 in [38], gives that the subfactor $N \rtimes_{\alpha} \mathbf{Z}_{n p} \subset M \rtimes_{\alpha} \mathbf{Z}_{n p}$ is strongly amenable. We have the same result for $\hat{\beta}$. Because the higher relative commutans of $N \rtimes_{\alpha} \mathbf{Z}_{n p} \subset M \rtimes_{\alpha} \mathbf{Z}_{n p}$ and $N \rtimes_{\beta} \mathbf{Z}_{n p} \subset M \rtimes_{\beta} \mathbf{Z}_{n p}$ are described by $p_{s}$ and $\gamma_{h}$, they are the same, and hence these two subfactors are isomorphic by Popa's classification theorem in [31].

It is easy to see that both $\hat{\alpha}$ and $\hat{\beta}$ have the same Loi invariant, so Popa's theorem in [33] gives outer conjugacy of $\hat{\alpha}$ and $\hat{\beta}$. With Takesaki duality [36], this gives the conclusion.

Q.E.D.

Take any non-strongly-outer $\alpha \in \operatorname{Aut}(M, N)$ with trivial Loi invariant. We call $\gamma_{h}(\alpha)$ an obstruction to flatness in the orbifold construction. (See [15] for a reason of this name.) If $\gamma_{h}(\alpha)=1$ for any such $\alpha \in \operatorname{Aut}(M, N)$, we then say that the obstruction for flatness in the orbifold construction vanishes for $N \subset M$. As a corollary to the above theorem, we get the following. We will see in Section 6 that we have several interesting subfactors satisfying the assumption in this Corollary.

Corollary 3.2 Let $N \subset M$ be a subfactor of the AFD type $I I_{1}$ factor with finite index and finite depth. Suppose that the obstruction for flatness in the orbifold construction vanishes for $N \subset M$. Then the triple $\left(p_{s}(\alpha), \nu_{\mathrm{alg}}(\alpha), \gamma_{h}(\alpha)\right)$ is a complete invariant of $\alpha \in \overline{\operatorname{Int}}(M, N)$ up to outer conjugacy.

For the range of the invariant, we have the following theorem.

Theorem 3.3 Let $N \subset M$ be a strongly amenable subfactor of type $I I_{1}$. Suppose that a non-strongly-outer $\sigma \in \operatorname{Aut}(M, N)$ with $\Phi(\sigma)=1$ and $\gamma_{h}(\sigma)=1$ is given and that the outer period $p_{o}(\sigma)$ of $\sigma$ is finite. Then for any positive integer $p$ and a pth root $\gamma$ of unity, there exists $\alpha \in \operatorname{Aut}(M, N)$ such that

$$
\left(p_{s}(\alpha), \nu_{\mathrm{alg}}(\alpha), \gamma_{h}(\alpha)\right)=(p, \sigma, \gamma)
$$


Proof We may assume $\gamma=1$, because then the general case follows from a tensoring the subfactor automorphism with $s_{p}^{\gamma}$ of Connes as above.

By Lemma 2.3, we may assume that $\sigma$ gives an outer action of $\mathbf{Z}_{n}$ with $n=p_{o}(\sigma)$. Let $P \subset Q$ be the orbifold subfactor $N \rtimes_{\sigma} \mathbf{Z}_{n} \subset M \rtimes_{\sigma} \mathbf{Z}_{n}$. We take a (unique) outer action $\theta$ of $\mathbf{Z}_{n p}$ on the AFD II factor $R$. (See [10].) Consider $(R \otimes P) \rtimes_{\theta \otimes \hat{\sigma}} \mathbf{Z}_{n p} \subset$ $(R \otimes Q) \rtimes_{\theta \otimes \hat{\sigma}} \mathbf{Z}_{n p}$. It is easy to see that this subfactor is isomorphic to $N \subset M$ by comparing the higher relative commutants, because the action $\theta \otimes \hat{\sigma}$ is strongly outer by [26, Corollary 7]. (Here we use an argument in the proof of Theorem 3.1.) We write $\alpha$ for the dual action $\widehat{\theta \otimes \hat{\sigma}}$. It is easy to see that $\alpha$ is an outer action of $\mathbf{Z}_{n p}$ and that $p_{s}(\alpha)=p, \nu_{\text {alg }}(\alpha)=\sigma, \gamma_{h}(\alpha)=1$.

Q.E.D.

\section{New invariants - an analytic approach -}

We next introduce another set of invariants defined analytically and identify them with those in Section 2 for strongly amenable subfactors.

Let $N \subset M$ be a subfactor of type $\mathrm{II}_{1}$. (We do not have to assume that the Jones index $[M: N]$ is finite here.) As in [22], we set

$$
\chi(M, N)=\frac{\operatorname{Ct}(M, N) \cap \overline{\operatorname{Int}}(M, N)}{\operatorname{Int}(M, N)},
$$

which is a subfactor analogue of the Connes invariant $\chi$ in [8].

Take any $\alpha \in \operatorname{Aut}(M, N)$. We define a relative asymptotic period $p_{a}(\alpha) \in \mathbf{N}$ by

$$
p_{a}(\alpha) \mathbf{Z}=\left\{n \in \mathbf{Z} \mid \alpha^{n} \in \mathrm{Ct}(M, N)\right\} .
$$

Suppose now $p_{a}(\alpha)>0$ and set $p=p_{a}(\alpha)$. The power $\alpha^{p}$ gives an element in $\operatorname{Ct}(M, N) / \operatorname{Int}(M, N)$. We write $\nu_{\text {ana }}(\alpha)$ for the orbit of the image of $\alpha^{p}$ under the action of $\operatorname{Aut}(M, N)$ given by $\alpha^{p} \mapsto \theta \cdot \alpha^{p} \cdot \theta^{-1}$ for $\theta \in \operatorname{Aut}(M, N)$ and call it an analytic $\nu$ invariant. This is an outer conjugacy invariant.

Suppose $\alpha \in \overline{\operatorname{Int}}(M, N)$ and $p=p_{a}(\alpha)>0$. Writing $\alpha=\operatorname{Ad} U$ with a unitary $U \in N^{\omega}$, we get $\alpha^{p}(U)=\lambda U$ with some $\lambda \in \mathbf{C}$ by the same argument as in $[24$, Section 2], which is a relative version of [5], [18]. (As usual, his $\lambda$ does not depend on choices of $U$ or a free ultrafilter $\omega$.) We set $\tilde{\kappa}(\alpha)=\lambda$ and call it a generalized relative Jones invariant. (The case in [24, Section 2] corresponds to the case $p=1$.) Note that in the original context of Jones [18], his $\kappa$ was a finer invariant of a factor than the Connes invariant $\chi$ in [8], but in our context, the invariant $\tilde{\kappa}$ is an invariant of an automorphism.

For a strongly amenable subfactor $N \subset M$ and $\alpha \in \overline{\operatorname{Int}}(M, N)$, we have two triplets of the invariants $\left(p_{s}(\alpha), \nu_{\text {alg }}(\alpha), \gamma_{h}(\alpha)\right)$ and $\left(p_{a}(\alpha), \nu_{\text {ana }}(\alpha), \tilde{\kappa}(\alpha)\right)$, and we now will compare these. We already know that $p_{s}(\alpha)=p_{a}(\alpha)$ and $\nu_{\text {alg }}(\alpha)=\nu_{\text {ana }}(\alpha)$. In our current framework, we get the following theorem easily for $\gamma_{h}$ and $\tilde{\kappa}$.

Theorem 4.1 For strongly amenable subfactors $N \subset M$ of type $I I_{1}$, we have $\overline{\gamma_{h}(\alpha)}=$ $\tilde{\kappa}(\alpha)$ for $\alpha \in \overline{\operatorname{Int}}(M, N)$. 
Proof Let $a \in M_{k}$ be a non-zero element with $\alpha^{p}(x) a=a x$ for all $x \in M$. Writing $\alpha=\operatorname{Ad} U$ with a unitary $U \in N^{\omega}$, we get $U a U^{*}=\gamma_{h}(\alpha) a$. (This is because the extension of $\alpha$ to the Jones tower is also given by $\operatorname{Ad} U$ by Loi's definition.) We also have $\alpha^{p}(U)=\tilde{\kappa}(\alpha) U$. These give

$$
\tilde{\kappa}(\alpha) U a=\alpha^{p}(U) a=a U=\overline{\gamma_{h}(\alpha)} U a,
$$

which gives the conclusion, because $\tilde{\kappa}(\alpha), \gamma_{h}(\alpha) \in \mathbf{C}$.

Q.E.D.

The above theorem was proved for the Hecke algebra subfactors of Wenzl [37] in the case $p_{a}(\alpha)=1$ in [24, Theorem 3.4] by a more complicated argument. V. F. R. Jones asked the author whether [24, Theorem 3.4] is valid for a general case or not, and the above theorem gives a positive answer. (Note that here we need a complex conjugate, which was unnecessary in [24] because the value of $\kappa$ there was \pm 1 .)

\section{The second classification theorem}

We next give another classification theorem of approximately inner automorphisms, which covers a different situation from the one in Theorem 3.1. This is based on the Connes technique given in [9, page 466]. (Also see [25, Theorem 20] and a remark at the end of [22].) Note that the case $p_{a}=0$ is covered by the relative version of the Connes classification theorem [7, Theorem 1] as in [27]. So we assume that $p_{a}>0$.

Theorem 5.1 Let $N \subset M$ be a subfactor of type $I I_{1}$ with the relative $M c D$ uff splitting property. Suppose that $\alpha, \beta \in \overline{\operatorname{Int}}(M, N)$ satisfying the following properties.

1. $p_{a}(\alpha)=p_{a}(\beta)=p>0$.

2. $\nu_{\text {ana }}(\alpha)=\nu_{\text {ana }}(\beta)$.

3. $\tilde{\kappa}(\alpha)=\tilde{\kappa}(\beta)$.

4. The outer period $n$ of $\alpha^{p}$ is finite.

5. $(n, p)=1$.

Then we have a unitary $u \in N$ and $\theta \in \overline{\operatorname{Int}}(M, N)$ such that $(\operatorname{Ad} u) \cdot \alpha=\theta \cdot \beta \cdot \theta^{-1}$.

Proof We may and do assume $\alpha^{p}=\beta^{p}$ modulo $\operatorname{Int}(M, N)$. Let $\sigma=\alpha^{p}$.

By 5 , there exist integers $k, m$ with $k n+m p=-1$. Then it is easy to see that

$$
p_{o}\left(\alpha^{p m+1}\right)=p_{a}\left(\alpha^{p m+1}\right)=p_{o}\left(\beta^{p m+1}\right)=p_{a}\left(\beta^{p m+1}\right)=p
$$

and $\alpha^{p m+1}, \beta^{p m+1} \in \overline{\operatorname{Int}}(M, N)$. As in the proof of lemma 2.3, we also get

$$
\gamma\left(\alpha^{p m+1}\right)=\gamma_{h}(\alpha)^{(p m+1)^{2}}=\gamma\left(\beta^{p m+1}\right) .
$$


This shows that there exist $\theta \in \overline{\operatorname{Int}}(M, N)$ and a unitary $u \in N$ with

$$
\theta \cdot \alpha \cdot \sigma^{m} \cdot \theta^{-1}=(\operatorname{Ad} u) \cdot \beta \cdot \sigma^{m},
$$

by the relative "translation" of [7, Theorem 2.3.1]. Because $\sigma^{m} \in \operatorname{Ct}(M, N)$ and $\theta^{-1} \in \overline{\operatorname{Int}}(M, N)$ commute modulo $\operatorname{Int}(M, N)$ as in [7, Lemma 2.2.2], we can conclude that there exists a unitary $v \in N$ with $\theta \cdot \alpha \cdot \theta^{-1}=(\operatorname{Ad} v) \cdot \beta$, which is the desired conclusion.

Q.E.D.

In this theorem, the finiteness condition $n$ is not so restrictive, because it holds automatically for subfactors of finite depth again. The condition $(n, p)=1$ is certainly more restrictive, and we do not know even whether the case $(n, p)>1$ can actually happens or not.

\section{Examples and remarks}

In this last section, we study the case $[M: N]<4$ as examples first. We then study Hecke algebra subfactors of Wenzl constructed in [37]. Finally, we discuss analogy between our classification here and the classification of automorphisms of injective type III factors in [9], [25], [35].

The subfactors with index less than 4 are completely classified by paragroups as in [28], [29], and we label them by the Dynkin diagrams $A_{n}, D_{2 n}, E_{6}$, and $E_{8}$. (See [1], [16], [17], [20], [34] for more details.) Our classification of automorphisms of these subfactors is complete except for the case of subfactors of type $A_{4 n-1}$ or $E_{6}$ with non-trivial $\nu(\alpha)$ and even $p_{a}(\alpha)$, as we shall see.

First recall that $\operatorname{Aut}(M, N)=\overline{\operatorname{Int}}(M, N)$ for $\mathrm{AFD}$ type $\mathrm{II}_{1}$ subfactors with principal graph $A_{n}, E_{6}$ or $E_{8}$ by [27]. The first example has already been treated in [22, Corollary 5.1].

Example 6.1 The following is a complete list of outer conjugacy classes automorphisms of AFD type $\mathrm{II}_{1}$ subfactors $N \subset M$ with principal graph $A_{2 n}$ of $E_{8}$.

$$
\begin{aligned}
& s_{0} \otimes \mathrm{id}, \\
& s_{p}^{\gamma} \otimes \mathrm{id}, \quad p>0, \gamma^{p}=1,
\end{aligned}
$$

where the tensor product factorization is with respect to the relative McDuff splitting $R \otimes N \subset R \otimes M$. The subfactor with principal graph $E_{8}^{(1)}$ also has this classification.

Example 6.2 Let $N \subset M$ be an AFD type $\mathrm{II}_{1}$ subfactor with principal graph $A_{4 n-3}$, $n>1$. Then as in [16] and [6, Theorem 2] (or [22, Proposition 4.4]), there exists an outer but not strongly outer automorphism $\sigma \in \operatorname{Aut}(M, N)$ of order 2 , which is unique up to $\operatorname{Int}(M, N)$. The following is a complete list of outer conjugacy classes automorphisms of $N \subset M$.

$$
\begin{array}{ll}
s_{0} \otimes \mathrm{id}, & \\
s_{p}^{\gamma} \otimes \mathrm{id}, & p>0, \gamma^{p}=1, \\
s_{p}^{\gamma} \otimes \theta_{p}, & p>0, \gamma^{p}=1,
\end{array}
$$


where the tensor product factorization is with respect to the relative McDuff splitting $R \otimes N \subset R \otimes M$, and $\theta_{p}$ is a " $p$ th root" of $\sigma$ constructed as in the proof of Theorem 3.3. The subfactor with principal graph $E_{7}^{(1)}$ also has this classification.

Example 6.3 Let $N \subset M$ be an AFD type $\mathrm{II}_{1}$ subfactor with principal graph $A_{4 n-1}$, $n>1$, or $E_{6}$. Then as in [16] and [6] (or [22]), there exists an outer but not strongly outer automorphism $\sigma \in \operatorname{Aut}(M, N)$ of order 2, which is unique up to $\operatorname{Int}(M, N)$. The following is a complete list of outer conjugacy classes automorphisms of $N \subset M$, except for the case of even $p_{a}$ and $p_{o}=2 p_{a}$.

$$
\begin{aligned}
& s_{0} \otimes \mathrm{id}, \\
& s_{p}^{\gamma} \otimes \mathrm{id}, \quad p>0, \gamma^{p}=1, \\
& s_{p}^{-\gamma} \otimes \sigma, \quad p \text { is odd }, \gamma^{p}=-1,
\end{aligned}
$$

where the tensor product factorization is with respect to the relative McDuff splitting $R \otimes N \subset R \otimes M$.

Example 6.4 In order to study the case excluded in the above Example more concretely, we study an outer action of $\mathbf{Z}_{4}$ on $N \subset M$ with principal graph $A_{2 m+1}$.

On one hand, Example 6.2 says that we have three different outer actions on $N \subset M$ with principal graph $A_{4 n-3}$ and these are all. They are listed as follows, where the graph $G_{1}$ denotes the co-standard graph of the action in the sense of Popa [32] and the graph $G_{2}$ the principal graph of the crossed product subfactor $N \rtimes \mathbf{Z}_{4} \subset M \rtimes \mathbf{Z}_{4}$.

\begin{tabular}{|l|l|l|}
\hline invariants & the graph $G_{1}$ & the graph $G_{2}$ \\
\hline$p_{a}=p_{o}=4$ & $A_{4 n-3}$ & $A_{4 n-3}$ \\
\hline$p_{a}=2, p_{o}=4, \gamma_{h}=-1$ & $D_{2 n}$ & $A_{4 n-3}$ \\
\hline$p_{a}=2, p_{o}=4, \gamma_{h}=1$ & $D_{2 n}$ & $D_{2 n}$ \\
\hline
\end{tabular}

On the other hand, if $p_{a}=2$ and $p_{o}=4$ on $N \subset M$ with principal graph $A_{4 n-1}$, then $\gamma_{h}^{2}=-1=\gamma$ by lemma 2.3. That is, we do not have an action of $\mathbf{Z}_{4}$. That is, we have only one outer action of $\mathbf{Z}_{4}, s_{4}^{1} \otimes \mathrm{id}$. (The same holds for any $\mathbf{Z}_{2 m}$ by the same reason.)

Next we also use the Loi invariant $\Phi(\alpha)$ for $\alpha \in \operatorname{Aut}(M, N)$ as in [27]. The following is a rather easy observation based on Popa's classification theorem [33].

Theorem 6.5 Suppose that $N \subset M$ is a strongly amenable subfactor of type $I I_{1}$ with $\operatorname{Ct}(M, N)=\operatorname{Int}(M, N)$. Then the triple $\left(p_{o}(\alpha), \gamma(\alpha), \Phi(\alpha)\right)$ is a complete invariant for outer conjugacy for $\alpha \in \operatorname{Aut}(M, N)$, where $p_{o}$ is an outer period.

All the combinations of $\left(p_{o}, \gamma, \Phi\right)$ with $\gamma^{p}=1(\gamma=1$ if $p=0)$ and $\Phi^{p}=1$ are realized. 
Proof The case $p_{o}=0$ is exactly follows from Popa's classification theorem in [33], so we assume $p_{o}>0$.

As in the proof of Theorem 3.1, we may assume that $\gamma=1$. Then $\alpha$ gives an outer action of $\mathbf{Z}_{p_{o}}$, thus Popa's classification theorem in [33] applies.

For the second half, let $\theta$ be the standard automorphism with given Loi invariant as in [27], [33]. Note that we have $\theta^{p}=1$. Then $s_{p}^{\gamma} \otimes \theta$ on the relative McDuff splitting gives the desired automorphism.

Q.E.D.

With this theorem, we have the following.

Example 6.6 Let $N \subset M$ be an AFD type $\mathrm{II}_{1}$ subfactor with principal graph $D_{2 n}$, $n>2$. Then as in [27] and [33], there exists a strongly outer automorphism $\theta \in$ $\operatorname{Aut}(M, N)$ of order 2 with non-trivial Loi invariant, which is unique up to $\operatorname{Int}(M, N)$. The following is a complete list of outer conjugacy classes automorphisms of $N \subset M$.

$$
\begin{aligned}
& s_{0} \otimes \mathrm{id}, \\
& s_{0} \otimes \theta, \\
& s_{p}^{\gamma} \otimes \mathrm{id}, \quad p>0, \gamma^{p}=1, \\
& s_{p}^{\gamma} \otimes \theta, \quad p \text { is even, } \gamma^{p}=1,
\end{aligned}
$$

where the tensor product factorization is with respect to the relative McDuff splitting $R \otimes N \subset R \otimes M$.

We next study the Hecke algebra subfactors of Wenzl [37]. (We use a description of them as quantum $S U(n)_{k}$ subfactors, which arise from the Wess-Zumino-Witten models $S U(n)_{k}$ as in [3].)

Example 6.7 First note that the $\nu$ invariant $\nu_{\text {alg }}(\alpha)$ (or $\nu_{\text {ana }}(\alpha)$ ) consists of a single element for the quantum $S U(n)_{k}$ subfactors for $n \geq 3$ and $k>n$. (Here we have to exclude the case $S U(3)_{3}$, for example. See [15].) Note that the Jones subfactors of type $A_{n}$ corresponds to $S U(2)_{n-1}$.

By [39], we have a large subclass of these subfactors where the obstruction to flatness in the orbifold construction vanishes. Because we always have finite depth for these cases, Corollary 3.2 applies, and we get a complete classification of $\overline{\operatorname{Int}}(M, N)$ up to outer conjugacy. In particular, we have this complete classification of approximately inner automorphisms for all the quantum subfactors $S U(2 n+1)_{k}$ with $n \geq 1$, $k>2 n+1$, because of Xu's result in [39], which has identified the obstruction to flatness in the orbifold construction with a conformal dimension in rational conformal field theory.

Furthermore, with a decsription of a paragroup in [11, Theorem 3.5] and a little bit of work, we can show that all the automorphisms of the quantum $S U(3)_{k}$ subfactors with $k>3$ are approximately inner. (It seems that this kind of argument would work for general quantum $S U(n)_{k}$ subfactors with $n>k$, but the graphs appearing in the paragroups are too complicated for the author to verify it in general.) Thus we have a complete classification of automorphisms of the quantum $S U(3)_{k}$ subfactors with $k>3$, up to outer conjugacy. 
At the end, we discuss analogy between our classification and a classification of automorphisms of injective type III factors. Because we are interested in approximately inner automorphisms, take the (unique) injective type $\mathrm{III}_{1}$ factor $M$, where any automorphism is approximately inner by [9, section 3.8], [25, Theorem 1]. We also have

$$
\operatorname{Ct}(M)=\left\{(\operatorname{Ad} u) \cdot \sigma_{t}^{\varphi} \mid u \text { is a unitary in } M, t \in \mathbf{R}\right\},
$$

where $\sigma_{t}^{\varphi}$ denotes the modular automorphism group (with respect to a weight $\varphi$ ), again by [9, section 3.8], [25, Theorem 1]. Then the classification of automorphisms of this $M$ is given as follows. Let $p_{a}(\alpha)$ be the asymptotic period of $\alpha$. If $p_{a}(\alpha)>0$, then $\alpha^{p}=($ Ad $u) \cdot \sigma_{t}^{\varphi}$ for some $u, t$. We define $\nu(\alpha)=t$ and call it the modular invariant. We can choose $\varphi$ to be a dominant weight with $\varphi \cdot \alpha=\varphi$, then $u \in M_{\varphi}$, the centralizer. The modular obstruction $\gamma_{m}(\alpha)$ is defined to be the scalar $\lambda$ with $\alpha(u)=\lambda u$. This is a well-defined outer conjugacy invariant of $\alpha$. We then have that the triple $\left(p_{a}(\alpha), \nu(\alpha), \gamma_{m}(\alpha)\right)$ is a complete invariant of $\operatorname{Aut}(M)$ up to outer conjugacy. This classification is essentially due to Connes [9], where he sketched only an outline. See [25] for the details.

It is clear that our classification here is conceptually parallel to this classification of automorphisms of injective type III factors. We, however, have something different for subfactors from the case of injective type III factors, that is, the obstruction to flatness in the orbifold construction may not vanish — as in [21], [39]. This causes combinatorial difficulty and gives a reason our classification here is not complete in

all the cases. We, however, still believe that our higher obstruction is the last missing invariant in the previous studies based on the above analogy.

\section{References}

[1] J. Bion-Nadal, Subfactor of the hyperfinite $I_{1}$ factor with Coxeter graph $E_{6}$ as invariant, J. Operator Theory 28 (1992), 27-50.

[2] D. Bisch, On the existence of central sequence in subfactors, Trans. Amer. Math. Soc. 321 (1990), 117-128.

[3] J. de Boer \& J. Goeree, Markov traces and $I I_{1}$ factors in conformal fields theory, Comm. Math. Phys. 139 (1991), 267-304.

[4] T. Ceccherini, Approximately inner and centrally free commuting squares of type $I I_{1}$ factors and their classification, to appear in J. Funct. Anal.

[5] J. Chen, The Connes invariant $\chi(M)$ and cohomology of groups, Ph.D. Dissertation, UC Berkeley, (1993).

[6] M. Choda \& H. Kosaki, Strongly outer actions for an inclusion of factors, J. Funct. Anal. 122 (1994), 315-332.

[7] A. Connes, Outer conjugacy classes of automorphisms of factors, Ann. Sci. ENS 8 (1975), 383-419. 
[8] A. Connes, Sur la classification des facteurs de type II, C. R. Acad. Sci. I 281 (1975), 13-15.

[9] A. Connes, On the classification of von Neumann algebras and their automorphisms, Symp. Math. XX (1976), 435-478.

[10] A. Connes, Periodic automorphisms of the hyperfinite factor of type $I I_{1}$, Acta Sci. Math. 39 (1977), 39-66.

[11] D. E. Evans \& Y. Kawahigashi, Orbifold subfactors from Hecke algebras, Comm. Math. Phys. 165 (1994), 445-484.

[12] D. E. Evans \& Y. Kawahigashi, Subfactors and conformal field theory, in "Quantum and non-commutative analysis", Kluwer Academic, (1993), 341-369.

[13] S. Goto, Orbifold construction for non-AFD subfactors, Internat. J. Math. 5 (1994), 725-746.

[14] S. Goto, Symmetric flat connections, triviality of Loi's invariant and orbifold subfactors, Publ. RIMS Kyoto Univ. 31 (1995), 609-624.

[15] S. Goto, Commutativity of automorphisms of subfactors modulo inner automorphisms, to appear in Proc. Amer. Math. Soc.

[16] M. Izumi, Application of fusion rules to classification of subfactors, Publ. RIMS Kyoto Univ. 27 (1991), 953-994.

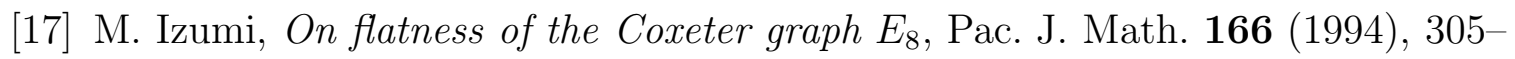
327.

[18] V. F. R. Jones, A factor anti-isomorphic to itself but without involutory antiautomorphisms, Math. Scand. 45 (1980), 103-117.

[19] V. F. R. Jones, Index for subfactors, Invent. Math. 72 (1983), 1-15.

[20] Y. Kawahigashi, Automorphisms commuting with a conditional expectation onto a subfactor with finite index, J. Operator Theory 28 (1992), 127-145.

[21] Y. Kawahigashi, On flatness of Ocneanu's connections on the Dynkin diagrams and classification of subfactors, J. Funct. Anal. 127 (1995), 63-107.

[22] Y. Kawahigashi, Centrally trivial automorphisms and an analogue of Connes's $\chi(M)$ for subfactors, Duke Math. J. 71 (1993), 93-118.

[23] Y. Kawahigashi, Classification of paragroup actions on subfactors, Publ. RIMS Kyoto Univ. 31 (1995), 481-517.

[24] Y. Kawahigashi, Orbifold subfactors, central sequences and the relative Jones invariant $\kappa$, Internat. Math. Res. Notices (1995), 129-140. 
[25] Y. Kawahigashi, C. E. Sutherland, \& M. Takesaki, The structure of the automorphism group of an injective factor and the cocycle conjugacy of discrete abelian group actions, Acta Math. 169 (1992), 105-130.

[26] H. Kosaki, Automorphisms in the irreducible decompositions of sectors, Quantum and non-commutative analysis, (ed. H. Araki et al.), Kluwer Academic, (1993), $305-316$.

[27] P. H. Loi, On automorphisms of subfactors, to appear in J. Funct. Anal.

[28] A. Ocneanu, Quantized group, string algebras and Galois theory for algebras, in "Operator algebras and applications, Vol. 2 (Warwick, 1987)," London Math. Soc. Lect. Note Series Vol. 136, Cambridge University Press, 1988, pp. 119-172.

[29] A. Ocneanu, "Quantum symmetry, differential geometry of finite graphs and classification of subfactors", University of Tokyo Seminary Notes 45, (Notes recorded by Y. Kawahigashi), 1991.

[30] M. Pimsner \& S. Popa, Iterating the basic constructions, Trans. Amer. Math. Soc. 310 (1988), 127-134.

[31] S. Popa, Classification of amenable subfactors of type II, Acta Math. 172 (1994), $352-445$.

[32] S. Popa, On the classification of actions of amenable groups on subfactors, C. R. Acad. Sc. Paris. 315 (1992), 295-299.

[33] S. Popa, Classification of actions of discrete amenable groups on amenable subfactors of type II, preprint 1992.

[34] V. S. Sunder \& A. K. Vijayarajan, On the non-occurrence of the Coxeter graphs $\beta_{2 n+1}, E_{7}, D_{2 n+1}$ as principal graphs of an inclusion of $I I_{1}$ factors, Pac. J. Math. 161 (1993), 185-200.

[35] C. E. Sutherland \& M. Takesaki, Actions of discrete amenable groups on injective factors of type $I I I_{\lambda}, \lambda \neq 1$, Pac. J. Math. 137 (1989), 405-444.

[36] M. Takesaki, Duality for crossed products and the structure of von Neumann algebras of type III, Acta Math. 131 (1973), 249-310.

[37] H. Wenzl, Hecke algebras of type $A$ and subfactors, Invent. Math. 92 (1988), $345-383$.

[38] C. Winsløw, Crossed products of $I I_{1}$ subfactors by strongly outer action, Proc. Amer. Math. Soc. 347 (1995), 985-991.

[39] F. Xu, Orbifold construction in subfactors, Comm. Math. Phys. 166 (1994), $237-254$.

[40] F. Xu, The flat parts of non-flat orbifolds, to appear in Pac. J. Math. 